\title{
Special Populations Networks
}

National Cancer Institute

\section{Source}

National Cancer Institute. Special Populations Networks. NCI Thesaurus. Code C19978.

$\mathrm{NCl}$ initiative to develop and maintain partnerships between scientific researchers and community leaders from a broad range of minority and underserved populations. 